\title{
Physical Layer Aspects of Wireless IoT
}

\author{
${ }^{\star}$ Shree Krishna Sharma ${ }^{+},{ }^{\star}$ Tadilo Endeshaw Bogale ${ }^{\dagger}$, Symeon Chatzinotas ${ }^{+}$, Xianbin Wang ${ }^{++}$and Long Bao Le ${ }^{\dagger}$ \\ SnT - securityandtrust.lu, University of Luxembourg, Luxembourg ${ }^{+}$ \\ Institute National de la Recherche Scientifique (INRS), Montréal, Canada ${ }^{\dagger}$ \\ University of Western Ontario, London, Canada ${ }^{++}$ \\ Email: \{shree.sharma, symeon.chatzinotas\}@uni.lu, \{tadilo.bogale,long.le\}@emt.inrs.ca, xianbin.wang@uwo.ca
} (Invited Paper)

\begin{abstract}
Internet of Things (IoT) is receiving important attention from industries and research communities as an important enabler for Fifth Generation (5G) of wireless communications. It has a wide range of application areas ranging from telecommunications to building smart communities, which will improve various aspects of our everyday lives. However, designing IoT-enabled wireless networks which can deliver a variety of services with desirable quality of experience under energy/resource constrained practical wireless scenarios is crucial. One of the important aspects here is to design the physical (PHY) layer of IoT-based wireless systems considering the practical constraints of energy efficiency, spectral efficiency, cost-effectiveness, and Quality of Service $(\mathrm{QoS})$. In this regard, this paper provides an overview of the related existing works from the PHY layer perspective. Various PHY layer enabling techniques are highlighted and the PHY layer aspects of different IoT enabled wireless networks are discussed. Finally, it provides some interesting research issues and future recommendations.
\end{abstract}

\section{INTRODUCTION}

Internet of Things (IoT) is a novel paradigm which is about connecting all types of physical objects (devices) to the Internet. The term IoT is also referred as Internet of Everything (IoE) [1], which basically brings people, data, processes, and things together in order to fulfill the everyday needs of people and society in an effective manner. Although there are other possibilities for communicating between IoT devices such as ethernet connectivity and power line communication, this paper focuses on wireless connectivity for IoT.

Currently, there are several parallel advances in different wireless technologies such as ZigBee, Bluetooth, Near Field Communication (NFC), Wi-Fi, cellular (GSM/3G/4G), Sigfox, Neul, LoRaWAN and Z-Wave [2], which have their own peculiar characteristics and application areas. IoT will potentially enable the integration of different wireless technologies and create market for new services. For example, the use of different wireless technologies such as GSM/3G/4G, NFC, Bluetooth, Wireless Local Area Network (WLAN), Global Positioning System (GPS) and sensor networks can be connected with SIM-card technology in order to support various services via a single device [3].

In wireless IoT environments, physical (PHY) layer of the protocol stack should be designed in such a way that energy efficiency, spectral efficiency, cost-effectiveness, and Quality

\footnotetext{
* Authors contribute equally.
}

of Service (QoS) requirements will be met with the help of cognitive (intelligent) interference management techniques and distributed resource allocation mechanisms [4]. Due to very high system dynamics, limited power and communication capabilities of the involved devices, robust PHY layer schemes which can ensure self-healing in worse-channel conditions are of significant importance. In this context, this paper provides a comprehensive overview on the PHY layer aspects of wireless IoT. Although there are papers in IoT from various aspects such as industrial perspective [5], Wireless Body Area Networks (WBANs) [6,7], Wireless Sensor Networks (WSNs) [8] and Satellite Communications (SatComs) [9], the contribution highlighting the key PHY layer issues is missing in the literature.

Besides, there are several emerging application areas of wireless IoT such as WBANs, WSNs, Machine type communication (Device to Device (D2D), Machine to Machine (M2M)), SatComs and 5G networks. Hence, it is significantly important to understand their specific PHY layer characteristics to devise cost-efficient as well as energy-efficient IoT transceivers. In this direction, first, we briefly discuss technical issues related to wireless IoT. Subsequently, we highlight the potential PHY layer enabling techniques for wireless IoT and provide an overview of the existing works in various IoT-enabled wireless networks. Finally, we conclude this paper by providing some interesting research issues and future recommendations.

\section{TeCHNicAl IsSues}

In contrast to other wireless communication paradigms, IoT has its own unique features and diverse requirements such as group-based communications, time-tolerant, small data transmission, secure connection, low cost and low energy consumption [9]. In IoT sensory environments, each sensor is mainly associated with sensing, processing and communication capabilities. Since many sensor types, manufacturers, protocols, etc., have to co-exist together in practice, the selected solution should be designed in such a way that its operation becomes independent of the device type and configuration. One of the main deployment challenges under such heterogeneous wireless environment is to provide reliable communication links to the low cost or/and power-constrained IoT devices. Another main problem is the high failure rate of the low-cost and low-powered sensor nodes [8]. In order 
TABLE I

MAIN REFERENCES FOR IOT IN VARIOUS WIRELESS NETWORKS

\begin{tabular}{l|l} 
Communication Types & References \\
\hline WBANs & {$[6,7]$} \\
WSNs & {$[8,12]$} \\
MTC (M2M, D2D, V2V) & {$[13-15,24,25]$} \\
SatCom & {$[9,16]$} \\
Cellular (LTE-A/5G) & {$[10,11,17-20]$} \\
\hline
\end{tabular}

to deploy these IoT sensors in practical scenarios, both the aspects of energy efficiency and transmission reliability are crucial.

Some of the main technical challenges in IoT are heterogeneity, connectivity, scaling, security, energy management, self-management capabilities, and standards [4]. The heterogeneity may exist in terms of devices, services and technologies. The device heterogeneity arises due to different capabilities of devices in terms of data rates and reliability, storage and computational power, mobility and compatibility with different technologies. In addition, several requirements such as bandwidth, reliability and latency of different existing services are highly diverse. In terms of connectivity, it is crucial to find out which devices need to be connected and which communication technology is suitable to connect them.

Furthermore, several other issues such as dynamic resource allocation, harmful interference mitigation and interoperability of different technologies have to be investigated while devising communication technologies for IoT. Besides the unreliable time-varying wireless channel and mobility related issues, the problem of interference becomes more severe as the number of devices increases in the wireless IoT context. Furthermore, as in other areas, energy management including harvesting, conservation and consumption, is another critical issue in order to design low-cost and energy-efficient wireless IoT systems. Moreover, the complexity of collecting, processing and combining information gathered via heterogeneous IoT devices as well as the amount of data required to make a reliable decision are continuously increasing over the time.

\section{Physical LAyer Aspects}

In this section, we provide PHY layer aspects of IoT in different application areas. In Table I, we list the important references, which discuss about the technical issues of IoT highlighting some of the PHY layer aspects in various types of wireless networks. Furthermore, Fig. 1 provides various enabling PHY layer techniques for wireless IoT, which are included in the following paragraphs in conjunction with the literature related to various wireless networks highlighted in Table I. As depicted in Fig. 1, the existing PHY layer protocols relevant to IoT are IEEE 802.15.4, IEEE 802.15.6, Bluetooth Low Energy (BLE), EPCgobal, LTE-A, Z-Wave, 6LowPAN, and NFC [7, 10].

\section{A. IoT in WBANs}

A WBAN comprises of in-body and on-body nodes which can continuously monitor a patient for the purposes of diagnosis and prescription. For data transmission, a WBAN uses licensed Wireless Medical Telemetry Services (WMTS),

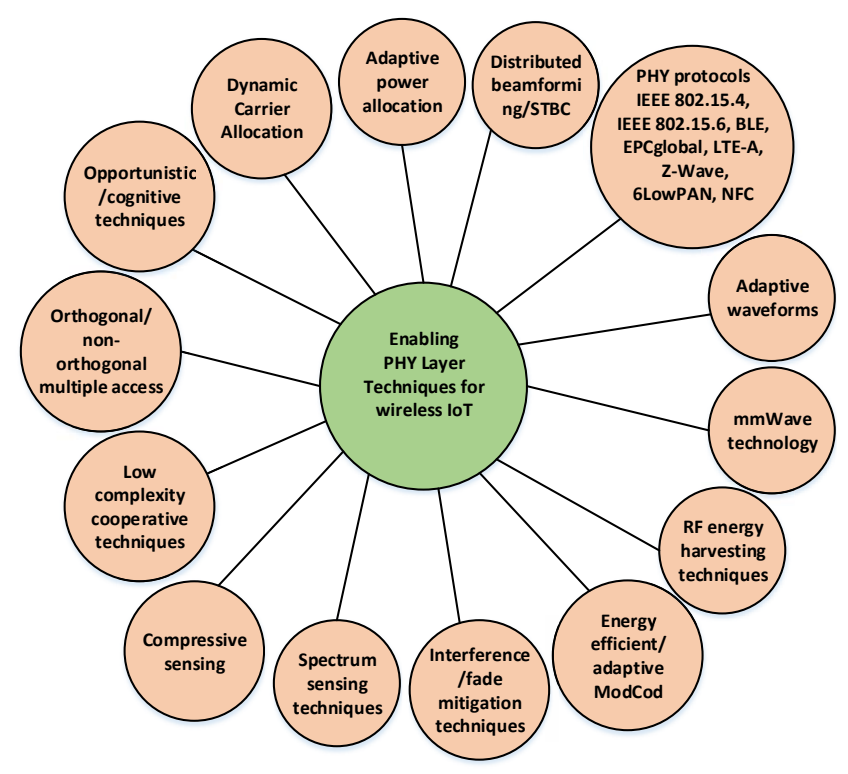

Fig. 1. Enabling PHY layer techniques for wireless IoT.

unlicensed ISM, Ultra-Wideband (UWB) and Medical Implant Communications Service (MICS) bands [6]. The licensed MICS band (402-405 MHz) is dedicated to the implant communication. An alternative spectrum for medical applications is $2.4 \mathrm{GHz}$ ISM band, however, this band is also used by other technologies, such as WiFi, Bluetooth, and Zigbee.

Two types of communication methods are mainly used for WBANs [6]: (i) beacon mode and (ii) non-beacon mode. Besides, the following three different tiers of communications are considered in WBANs [6]: (i) Intra-WBAN communication (Tier 1) which involves the interaction of nodes and their respective transmission ranges (about 2 meters) in and around the human body, (ii) Inter-WBAN communication (Tier 2) which is considered in between the personal server in the first tier and one or more access points and aims to interconnect a WBAN with several networks such as cellular and Internet, and (iii) Beyond-WBAN Communication (Tier 3) which is more application-specific such as the connection from Internet to a medical server via a gateway device.

Various PHY layer communication aspects of WBANs such as antenna design, electromagnetic coupling, radio frequency communications, and signal propagation have been detailed in $[6,7]$. The PHY layer in WBANs involves the transformation of PHY Layer Service Data Unit (PSDU) into a PHY Layer Protocol Data Unit (PPDU). In this regard, IEEE 802.15.6 standard has provided the following three different types of PHY layer specifications in WBANs: (i) human body communication, (ii) narrow band, and (iii) UWB.

\section{B. IoT in WSNs}

The PHY layer techniques for WSNs are mainly designed with the objectives of achieving high diversity gains, maximizing energy efficiency and reducing the computational burden. In this direction, the main research aspects can be categorized into [8]: (i) techniques for energy-efficient and reliable transceiver design; and ii) techniques for low complex- 
ity data fusion rules. The energy-efficient transceiver design techniques involve spatial diversity techniques such as Space Time Block Code (STBC) and beamforming considering either multiple antennas or a single antenna at each sensor node and energy-efficient modulation and detection techniques. Since it is practically difficult to equip all the sensor nodes with multiple antennas, most of the works consider distributed antenna system and employ STBC or/and beamforming in a distributed manner. In the distributed approach, maintaining the global synchronization (carrier frequency, time/phase) over the considered WSN is a crucial aspect. Although STBC is relatively robust to phase offset, the performance of distributed beamforming is affected with the phase mismatch. Besides, phase or/and synchronization consumes a significant amount of energy [8].

For short distance transmission which is most probable in IoT sensory environments, the circuit energy consumption mainly dominates the transmission energy [12]. In this scenario, it is important to design energy-efficient approaches by optimizing modulation constellations and/or transmission durations. It is worthy to mention that there exists a limit for transmission distance beyond which no energy saving gain can be achieved by optimizing the modulation sizes [12]. The tight energy margin used with the objective of reducing power consumption may cause degradation in the symbol error rate. Besides, energy consumption at the node increases when it has to retransmit due to transmission failure caused either due to high symbol error or collision. In the case of low complexity sensor data fusion, the main objective becomes to reduce the network overhead while guaranteeing the desired estimation/detection performance.

Moreover, the sensing capability of a sensor represents its ability to provide a certain quality of information to a particular task depending on the employed application. Sensing techniques may range from simple energy detection to the complex eigenvalue based detection [21]. Besides, cooperative sensing techniques have been recently investigated in order to address the hidden node problem in WSNs comprising of heterogeneous nodes [22]. In large WSNs, IoT sensor selection and searching is a critical issue and new approaches need to be investigated since traditional text-based search approaches are not accurate to capture the main sensor characteristics [23].

\section{IoT in Machine Type Communications (MTCs)}

Future IoT enabled wireless networks are expected to incorporate a massive number of connected devices which may reach up to 100 times compared to the current wireless networks. The potential use cases in this regard are wearable devices, MTCs (M2M, D2D, V2V) and wireless sensors [13, 14,24]. These applications promote direct communication between nodes without transmitting data to the base stations in cellular systems, which could help to bring significant performance improvement in terms of both spectrum and energy efficiency [15]. As most of such applications are likely real time and delay sensitive, latency may also need to be examined in addition to the PHY layer throughput which suggests to dynamically monitor the radio resources at the cross layer.

Most MTCs rely on IEEE 802.11p which employs carrier sense multiple access with collision avoidance. This is a reasonable solution since it inherently enables a decentralized allocation of resources. However, as such an approach depends on the sensing performance at the transmitter, it suffers from a hidden terminal problem which may lead to severe performance degradation. To address this problem, various conventional orthogonal and Non-Orthogonal Multiple Access (NOMA) techniques have been proposed [25]. Although these techniques are simple to realize, they may not be viable solutions for the future dense MTC. In this aspect, it is desirable to leverage spectrum sensing algorithms of cognitive radio to mitigate the hidden terminal problem [14,21].

\section{IoT in Satellite Communications}

SatCom plays a vital role in delivering broadband services over a wide area and is the viable option to cover a number of sectors such as land mobile, maritime, aeronautical, transport, military, rescue and disaster relief. In many scenarios, sensors/actuators are distributed over a very wide area and in some cases, they are located in remote areas which are not accessible by the terrestrial networks. In these scenarios, Internet of Remote Things (IoRT) paradigm can be realized using SatCom, especially for smart grid, environmental monitoring and emergency management [9]. Authors in [26] carried out a feasibility study on the use of Low Earth Orbit (LEO) satellite constellation for distributed control and automation in a smart grid scenario while considering the strict latency requirements. The key parameters which affect network management performance in such LEO networks are latency, packet loss due to congestion, bandwidth, packet corruption due to transmission errors, and orbital dynamics. Furthermore, authors in [16] have investigated the performance of the satellite-based M2M communication by employing a clustering mechanisms and multiple satellite gateways. Recently, there is an increasing interest in low cost small satellites in LEO such as nanosatellites and CubeSats, leading to the concept of Big LEO.

The characteristics of satellite channels vary depending on the weather, and the rain fading heavily affects the performance of satellite based IoT system [9]. Besides, there may exist several interfering sources such as cochannel satellite and cochannel terrestrial systems. In order to address above issues, suitable rain mitigation techniques, dynamic carrier as well as power allocation schemes need to be investigated. In order to address the spectrum scarcity problem, the concept of cognitive radio is getting an important attention in SatCom [27]. Various techniques such as beamforming, power allocation, and carrier allocation have been studied considering the coexistence of satellite and terrestrial systems [28] and two satellite systems [29].

\section{E. IoT in LTE-Advanced/5G Networks}

The rapidly increasing requirements of IoT applications in 5G systems demand massive QoS service enhancements in terms of spectrum efficiency, energy efficiency, connectivity 
and latency. To meet these diverse requirements, an efficient, scalable and flexible air-interface is required. Therefore, different modules of PHY and MAC layers should be optimized so that they can be configured flexibly according to the technical specifications of each use case. It is also almost clear that the IoT enabled systems may need to exploit the underutilized millimeter wave (mmWave) systems due to the availability of significant bandwidth (e.g., around $12.9 \mathrm{GHz}$ in the E-band [32]). Besides, mmWave systems employ massive antenna array technologies (i.e., massive Multiple Input Multiple Output (MIMO)) where their performance depends on the beamforming and multiple access strategies. For this reason, the future wireless IoT devices may utilize the recently developed hybrid analog-digital beamforming and multiple access such as NOMA techniques [11,19].

The design of optimal transmission waveform is one of the crucial steps to satisfy the requirements of the future $5 \mathrm{G}$ networks. Although several approaches are utilized in earlier generations, OFDM is chosen by a number of recent wireless standards as it has many advantages compared to TDMA, FDMA and CDMA counterparts [30]. For multiuser systems, such as LTE-A, intercell-interference which is created among sub-carriers is one of the major problem especially for cell edge users. To address this, different solutions have been suggested including user scheduling, combining the benefits of different techniques such as OFDM and CDMA [38].

Another disadvantage of OFDM transmission is its high Peak to Average Power Ratio (PAPR) of the transmitted waveform, especially when the block length is large. This will consequently require high quality power amplifier which is often expensive, especially for terminals with low transmission power capabilities. Furthermore, high PAPR results in serious degradation of bit error rate performance [31]. To this end, employing different modified OFDM transmissions such as single carrier and generalized OFDM could be the most promising solutions $[18,20]$.

The IoT-based $5 \mathrm{G}$ networks will also be deployed at the microwave frequency ranges where there is a spectrum crunch. For these spectrum regions, the current transmission techniques employ a Nyquist transmission approach where only one information symbol is incorporated per symbol duration. Since future $5 \mathrm{G}$ networks require significant capacity, recent literature suggests a transmission strategy which can handle more information content per symbol period. In this respect, faster than Nyquist method is motivated to enable data rate beyond what is defined by the Nyquist criterion without imposing additional bandwidth which can be one of the potential candidates for the future IoT applications [33].

\section{Challenges and Future Recommendations}

In the previous sections, we have highlighted some of the design considerations and potential use cases of the wireless IoT. In fact, this new communication paradigm will bring a number of technical challenges at several layers of protocol stack. In the following, we provide some of these challenges and future recommendations mainly at the PHY layer.
1. Low-cost energy-efficient transceiver: In general, a massive amount of IoT devices may need to be fabricated in a cost effective manner. Furthermore, these devices will most likely be battery operated and located in a remote area where charging may be economically infeasible. On the other hand, the IoT devices may likely be miniaturized in size and nonreplaceable. This implies that cost, energy, network lifetime and space efficiency will be the critical challenges. In this regard, different signal processing tools from WSN and radar applications can be adopted for IoT-based MTC communication applications [34].

2. Hardware imperfections: The future IoT-enabled wireless systems may need to operate in a non-orthogonal transmission scheme in which receivers may need to have interference mitigation capabilities. This can be enabled by accurately learning the interference channel and its corresponding covariance matrix, which are estimated and often imperfect. One main possible cause is the limited dynamic range of the Analog to Digital Converter (ADC), especially for low cost devices [36]. In this regard, the design of a non-orthogonal transmission with low resolution ADCs is an interesting research area.

3. Compressive signal processing for massive IoT systems: While considering wideband spectrum utilization, Nyquistbased sampling is not feasible due to the requirements of very high rate ADC [39]. In this regard, it is an interesting research direction to exploit the inherent time, frequency sparsity caused by sporadic traffic of IoT based systems as well as spatial sparsity exhibited due to multipath environment and to apply compressive signal processing in order to devise efficient wideband sensing and channel estimation techniques. 4. Spectrum management for Wireless IoT: The future IoT applications require a highly scalable, reliable and available radio spectrum. The existing spectrum allocation strategies which mainly adopt orthogonalization may not be viable solutions. In this regard, dynamic and non-orthogonal spectrum allocation policies are promising. One possible direction could be to allow the IoT user to simultaneously utilize both microwave and mmWave carrier frequency bands (i.e., dual band connectivity) [11]. On the other hand, to support wideband IoT applications, both contiguous and non-contiguous carrier aggregation can be employed especially at the microwave frequency bands [35]. In this context, the main challenge is how to efficiently realize wideband IoT systems which are capable of reaping the benefits of both microwave and mmWave frequency bands.

5. Cross layer design for end to end system reliability: The PHY layer parameters should be effectively utilized in devising MAC layer and network layer protocols in order to design end to end reliable communication systems. For example, in wireless energy harvesting applications, dynamic duty cycles create challenges for MAC layer protocol design in terms of various aspects such as synchronization, reliability and resource utilization efficiency. To address this, solutions for duty cycle-aware middle-ware between MAC and PHY layer power management is highly desirable [37]. Moreover, dynamic duty cycling has a considerable impact on the end 
to end performance of the network layer in terms of delay and throughput. In this regard, the design of duty cycle-aware routing protocol is a promising research direction.

6. Low complexity cooperative techniques: Cooperation among IoT sensors is important to achieve reliable communication with low power in severe fading conditions. Among several possible cooperative techniques, cooperative channel coding can be considered as one promising PHY layer approach for IoT-based systems. To this end, investigation of an effective channel coding with flexible packet length and low complexity while providing a good link quality is an interesting research problem.

\section{CONCLUSions}

IoT being a complex paradigm, it faces several technical challenges in wireless communications which need to be addressed with further research and development activities. This paper first highlighted the key PHY layer techniques for enabling wireless IoT. Then, the paper has provided an overview on PHY layer aspects of IoT in different wireless networks such as WBANs, WSNs, MTC, SatComs, and cellular networks. It has been suggested that future research efforts should be focused in designing low-cost and energy-efficient transceivers and in incorporating PHY layer parameters in the design of MAC and network layer protocols, to realize a reliable IoT-based wireless system.

\section{REFERENCES}

[1] CISCO, "CISCO visual networking index: Global mobile data traffic forecast update, 2015-2020," White paper, Feb. 2016.

[2] RS Components, "11 Internet of Things (IoT) Protocols You Need to Know About," available online: http://www.rsonline.com/designspark/electronics/knowledge-item/eleven-internetof-things-iot-protocols-you-need-to-know-about.

[3] H. Sundmaeker, et. al., "Vision and challenges for realising the Internet of Things," Pub. Office EU, Mar. 2010.

[4] M. Zorzi, et. al., "From today's intranet of things to a future Internet of Things: a wireless- and mobility-related view," IEEE Wireless Commun., vol. 17, no. 6, pp. 44-51, Dec. 2010.

[5] C. Perera, et. al., "A survey on Internet of Things from industrial market perspective," IEEE Access, vol. 2, pp. 1660-1679, 2014.

[6] S. Movassaghi, et. al., "Wireless body area networks: A survey," IEEE Commun. Surveys Tut., vol. 16, no. 3, pp. 1658-1686, Third 2014.

[7] S. Ullah, et. al., "A comprehensive survey of wireless body area networks," J. Med. Syst., vol. 36, no. 1065, pp. 1065-1094, June 2012.

[8] T. Kim, et. al., "Physical layer and medium access control design in energy efficient sensor networks: An overview," IEEE Trans. Industrial Inform., vol. 11, no. 1, pp. 2-15, Feb. 2015.

[9] M. De Sanctis, et. al., "Satellite communications supporting Internet of remote things," IEEE Internet of Things J., vol. 3, no. 1, pp. 113-123, Feb. 2016.

[10] A. Al-Fuqaha, et. al., "Internet of Things: A survey on enabling technologies, protocols, and applications," IEEE Commun. Surveys Tut., vol. 17, no. 4, pp. 2347-2376, Fourthquarter 2015.

[11] T. E. Bogale and L. B. Le, "Massive MIMO and mmWave for 5G Wireless HetNet: Potential Benefits and Challenges," IEEE Veh. Technol. Mag., vol. 11, no. 1, pp. 64-75, 2016.

[12] Shuguang Cui, A. J. Goldsmith, and A. Bahai, "Energy-constrained modulation optimization," IEEE Trans. Wireless Commun., vol. 4, no. 5, pp. 2349-2360, Sept 2005.

[13] M. Hasan, E. Hossain, and D. Niyato, "Random access for machineto-machine communication in LTE-advanced networks: issues and approaches," IEEE Commun. Mag., vol. 51, no. 6, pp. 86-93, June 2013.

[14] A. Aijaz and A. H. Aghvami, "Cognitive machine-to-machine communications for Internet-of-Things: A protocol stack perspective," IEEE Internet of Things J., vol. 2, no. 2, pp. 103-112, Apr. 2015.
[15] W. Wang and V. K. N. Lau, "Delay-aware cross-layer design for deviceto-device communications in future cellular systems," IEEE Commun. Mag., vol. 52, no. 6, pp. 133-139, June 2014.

[16] S. Vassaki, et. al., "Satellite-based sensor networks: M2M sensor communications and connectivity analysis," in Int. Conf. Telecommun. and Multimedia, July 2014, pp. 132-137.

[17] J. M. Liang, et. al., "An energy-efficient sleep scheduling with QoS consideration in 3GPP LTE-advanced networks for internet of things," IEEE J. on Emerging and Sel. Topics in Circuits and Systems, vol. 3, no. 1, pp. 13-22, Mar. 2013.

[18] F. Schaich, T. Wild, and Y. Chen, "Waveform contenders for 5G suitability for short packet and low latency transmissions," in IEEE VTC Spring, May 2014, pp. 1-5.

[19] B. Wang, et. al., "Comparison study of non-orthogonal multiple access schemes for 5G," in 2015 IEEE Int. Symp. Broadband Multimedia Systems and Broadcast., June 2015, pp. 1-5.

[20] X. Wang, et. al., "OFDMA-based channel-width adaptation in wireless mesh networks," IEEE Trans. Veh. Technol., vol. 63, no. 8, pp. 40394052, Oct. 2014.

[21] S. K. Sharma, et. al., "Cognitive radio techniques under practical imperfections: A survey," IEEE Commun. Surveys Tut., vol. 17, no. 4, pp. 1858-1884, Fourthquarter 2015.

[22] S. K. Sharma, S. Chatzinotas, and Björn Ottersten, "Cooperative Spectrum Sensing for Heterogeneous Sensor Networks Using Multiple Decision Statistics", Int. Conf. CROWNCOM, Apr. 2015, pp. 321-333.

[23] C. Perera, et. al., "Sensor search techniques for sensing as a service architecture for the internet of things," IEEE Sensors J., vol. 14, no. 2, pp. 406-420, Feb. 2014

[24] A. Laya, et. al., "Device-to-device communications and small cells: Enabling spectrum reuse for dense networks," IEEE Commun. Mag., pp. 98 - 105, Aug. 2014.

[25] J. Blum and A. Eskandarian, "A reliable link-layer protocol for robust and scalable intervehicle communications," IEEE Trans. Intell. Transp. Syst., vol. 8, no. 1, pp. 413, Feb. 2013.

[26] Q. Yang, et. al., "On the use of LEO satellite constellation for active network management in power distribution networks," IEEE Trans. Smart Grid, vol. 3, no. 3, pp. 1371-1381, Sept. 2012.

[27] S. K. Sharma, S. Chatzinotas, and B. Ottersten, "Cognitive radio techniques for satellite communication systems," in IEEE VTC Fall, Sept. 2013, pp. 1-5.

[28] E. Lagunas, et. al., "Resource allocation for cognitive satellite communications with incumbent terrestrial networks," IEEE Trans. Cognitive Commun. and Networking, vol. 1, no. 3, pp. 305-317, Sept. 2015.

[29] S. K. Sharma, S. Chatzinotas, and B. Ottersten, "Inline Interference Mitigation Techniques for Spectral Coexistence of GEO and NGEO Satellites", Int. J. Satellite Commun. and Networking, Sept. 2014.

[30] J. G. Andrews, et. al., "What will 5G be?," IEEE Sel. Area Commun., vol. 32, no. 6, pp. 1065 - 1082, Jun. 2014.

[31] J. C. Chen and C. P. Li, "Tone reservation using near-optimal peak reduction tone set selection algorithm for PAPR reduction in OFDM systems," IEEE Signal Process. Letters, vol. 17, no. 11, pp. 933 - 936, Nov. 2010.

[32] Z. Pi and F. Khan, "An introduction to millimeter-wave mobile broadband systems," IEEE Commun. Mag., pp. 101 - 107, Jun. 2011.

[33] T. E. Bogale, et. al., "Orthogonal faster than Nyquist for SIMO wireless systems," to appear in IEEE Globecom, 2016.

[34] O. Demigha, W. K. Hidouci, and T. Ahmed, "On energy efficiency in collaborative target tracking in wireless sensor network: A review," IEEE Commun. Surveys Tut., vol. 15, no. 3, pp. 1210-1222, Third 2013.

[35] Z. Khan, et. al., "Carrier aggregation/channel bonding in next generation cellular networks: methods and challenges," IEEE Network, vol. 28, no. 6, pp. 34-40, Nov 2014.

[36] F. M. L. Tavares, et. al., "On the impact of receiver imperfections on the MMSE-IRC receiver performance in 5G networks," in IEEE VTC Spring, May 2014, pp. 1-6.

[37] P. Kamalinejad, et. al., "Wireless energy harvesting for the Internet of Things," IEEE Commun. Mag., vol. 53, no. 6, pp. 102-108, June 2015.

[38] B. Jiao, et. al., "A combination of CS-CDMA and OFDM for enhanced LTE on downlink channel," Journal of Communications and Networks, vol. 15, no. 1, pp. 8-14, Feb. 2013.

[39] S. K. Sharma, et. al., "Application of Compressive Sensing in Cognitive Radio Communications: A Survey," IEEE Commun. Surveys Tut., vol. 18, no. 3, pp. 1838-1860, thirdquarter 2016. 